SUSTENERE

Publishing Corporation

\title{
NEUROCIÊNCIA NA EDUCAÇÃO: CAUSAS, CONSEQUÊNCIAS E INTERVENÇÕES CORRETIVAS PARA DISGRAFIA
}

\section{RESUMO}

Este trabalho aborda as principais descobertas da Neurociência relacionadas ao desenvolvimento da linguagem escrita e sua relação com a aprendizagem,

buscando evidenciar os conceitos, mecanismos de avaliação e intervenção corretiva da caligrafia em casos de disgrafia.

PALAVRAS-CHAVES: Neurociência na Educação; Linguagem Escrita; Disgrafia.

\section{NEUROSCIENCE IN EDUCATION: CAUSES, CONSEQUENCES AND CORRECTIVE INTERVENTIONS FOR DYSGRAPHIA}

\section{ABSTRACT}

This paper addresses the major neuroscience findings related to the development of written language and its relation to learning, disclosing the concepts, evaluation mechanisms and corrective intervention calligraphy in cases of dysgraphia.

KEYWORDS: Neuroscience in Education; Written Language; Dysgraphia.

Scire Salutis, Aquidabã, v.5, n.1, Out, Nov, Dez 2014, Jan, Fev, Mar, Abr, Mai, Jun, Jul, Ago, Set, Out 2015.

ISSN 2236-9600

\section{SECTION: Articles}

TOPIC: Enfermagem

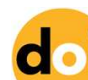

DOI: 10.6008/SPC2236-9600.2015.001.0003

Rogério Reis Benedito Universidade Federal de Lavras, Brasil http://lattes.cnpq.br/5955731181783562 rogerioages@infonet.com.br

Hugo Márcio Rodrigues de Almeida Universidade de Aveiro, Portugal http://lattes.cnpq.br/6270545294068170

Received: 05/02/2015

Approved: $13 / 09 / 2015$

Reviewed anonymously in the process of blind peer.

Referencing this:

BENEDITO, R. R.; ALMEIDA, H. M. R.. Neurociência na educação: causas, consequências e intervenções corretivas para disgrafia. Scire Salutis, Aquidabã, v.5, n.2, p.24-36, 2015. DOI:

http://dx.doi.org/10.6008/SPC22369600.2015 .001 .0003 


\section{INTRODUÇÃO}

As descobertas das Neurociências podem permitir uma revisão das práxis educativas e, isso, constituiria um fator imperativo para se vislumbrar um ensino orientado à forma como os aprendizes constroem o seu conhecimento. Logo, é necessário ampliar a compreensão sobre os fenômenos relacionados à aprendizagem, principalmente no que se refere à identificação de causas de déficits de aprendizagem, corroborando com essa ideia a Royal Society da Inglaterra, publicou em 2011 um documento de políticas intitulado 'Neuroscience: implications for education and lifelong learning' em que os autores argumentaram que havia uma distância entre as neurociências e a educação, o que sugeririam a possibilidade de uma mudança nas práticas educacionais impulsionadas por novas descobertas científicas assim como houve com as práticas médicas há cerca de um século.

Este trabalho propõe-se a realizar uma revisão sistemática sobre Neurociências e aprendizagem, a fim de se evidenciar descobertas científicas acerca do desenvolvimento da linguagem escrita com foco em fatores causais, consequências para aprendizagem e remediação da grafia relacionados à disgrafia, estabelecendo uma relação entre a identificação e avaliação e as possibilidades de intervenções para ajuste e minoração dos efeitos dos distúrbios de caligrafia nos primeiros anos de formação escolar.

Deve-se ainda considerar o distanciamento e, em contraste, o entusiasmo de educadores e demais profissionais da educação com este corpo do saber, em parte pela ausência em quantidade, de estudos significativos por parte da ciência educacional que considerem as recentes descobertas das Neurociências.

\section{METODOLOGIA}

A pesquisa identificou trinta e seis artigos, no período de abril a junho de 2015. Empregouse como motor de busca o Web off Sciene (http://apps.webofknowledge.com/) indexador de bases de dados bibliográficos, utilizando as palavras-chave "Educational Neuroscience", "Writing Language Acquisition", e "Dysgraphia" em associação. Inicialmente apenas doze artigos publicados em língua inglesa cujo periódico possuísse fator de impacto igual ou superior a 1,9 e o texto integral do artigo estivesse disponível para download de forma gratuita foram considerados e selecionados. Quando encontradas referências adicionais relevantes em artigos identificados na busca, estas também foram incluídas na revisão. Ao final totalizou-se um quantitativo de vinte e dois artigos sendo doze selecionados na busca inicial e mais dez incluídos a partir das referências, inclusão esta que, também obedeceu ao mesmo critério para o idioma do artigo e fator de impacto do periódico. Assim, considerou-se artigos que tratam da aquisição de linguagem, de distúrbios específicos de aprendizagem ligados a disgrafia (ou seja, que vão desde os componentes básicos da psicogênese da linguagem escrita, tais como os fatores ambientais e neurobiológicos envolvidos no desenvolvimento da linguagem escrita até a identificação de desordens relacionadas à aquisição 
da escrita com foco na disgrafia). Embora a escrita seja um movimento baseada em processos linguísticos, na integração viso-motora e algumas pessoas disgráficas possam apresentar outros distúrbios de aprendizagem, a pesquisa concentrou esforço na disgrafia como foco do estudo.

\section{REVISÃO TEÓRICA}

\section{Linguagem e aprendizagem}

A aprendizagem é um fenômeno cujas variáveis ainda não são conhecidas em todos os seus aspectos, no entanto pode-se inferir que a linguagem é fator determinante no processo de aprendizagem, haja vista que a comunicação é tida como essencial à aquisição de novos conhecimentos e a manifestação expressa do que já se aprendeu. Assim, na concepção de (JACKENDOFF, 1994) "A linguagem pode ser definida como um conhecimento abstrato, internalizado que é a base para a comunicação e funciona como uma janela do pensamento". A construção do sujeito social dá-se a partir das relações estabelecidas entre indivíduos e circunstâncias, tendo como elo fundamental a linguagem em todas as suas manifestações possíveis.

Ao lançar mão de recursos empíricos aliados a uma análise do referencial teórico utilizado para este estudo permitiu-se ponderar sobre a qualidade da comunicação e, com isso, notar a relação entre estes dois fatores, a ponto de a linguagem influenciar a qualidade da aprendizagem, o que impõe a necessidade de se conhecer de forma mais aprofundada os conceitos e mecanismos inerentes à linguagem e sua relação com a aprendizagem, e isso pode ser sustentado na afirmação de (MARKMAN et al., 2011) de que a "A linguagem fornece as ferramentas para nos revelar aos outros no estabelecimento e manutenção de relacionamentos e impulsiona o aprendizado perceptual que contribui para a cognição." Vemos, então que a linguagem, além de ser uma forma de expressar a si mesmo, também é uma poderosa ferramenta de construção da subjetividade do indivíduo, possibilitando que este crie uma estrutura acessível de seus conhecimentos.

\section{A Importância da Escrita no Cotidiano Escolar}

Hoje a evolução tecnológica possibilita o uso de ferramentas de comunicação capazes de converter, por exemplo, a fala em texto digitalizado, no entanto, a escrita manual ainda é a forma mais imediata e a mais utilizada no âmbito escolar para o registro e avaliação dos conhecimentos trabalhados pelos alunos. A qualidade do desempenho no uso da escrita exerce influência considerável na autoimagem do estudante como tal, já que a mesma também é a base para a construção de outras competências valorizadas no universo escolar. (FEDER \& MAJNEMER, 2007), em seu estudo, reforçam esse entendimento quando citam que "O desenvolvimento da capacidade de escrita não só é importante na construção de autoestima de uma criança, como 
também é considerado um ingrediente essencial para o sucesso na escola.". Qualitativamente, fica evidente a importância da escrita no contexto escolar, quantitativamente pode-se argumentar que as atividades que envolvem a motricidade fina, como a escrita ocupam um tempo considerável no dia-a-dia da escola como podemos ver, "As crianças passam $31-60 \%$ do seu dia escolar realizando escrita e outras tarefas motoras finas, e dificuldades nesta área podem interferir com o rendimento escolar" (FEDER \& MAJNEMER, 2007). Além disso, a letra ilegível pode, ainda criar outros obstáculos ao desenvolvimento escolar, segundo (FEDER \& MAJNEMER, 2007) "Caligrafia ilegível pode criar uma barreira para o desenvolvimento de outras habilidades de ordem superior, como ortografia e composição de história. Apesar do uso de computadores, a escrita à mão continua a ser uma habilidade de desenvolvimento importante a ser dominada por uma criança.". A escrita, assim como outros aspectos da língua é de extrema importância em nossa sociedade, uma série de afazeres diários, como preencher um cheque ou escrever um bilhete tornar-se-iam inexequíveis sem o domínio da escrita.

\section{Problemas de Escrita e suas Consequências}

Os problemas de escrita são bastante comuns, atingindo um número considerável de crianças como podemos ver em (FEDER \& MAJNEMER, 2007) "As estimativas de disfunção de escrita em crianças variam de 10 a $30 \%$ e as consequências para o desempenho acadêmico têm sido bem documentadas.". Na realidade educacional brasileira a percepção desses índices ainda causa pouca mobilização para estudos e propostas de políticas de intervenção que objetivem atenuar os efeitos dos problemas de escrita.

Apesar da pouca atenção e importância ainda dada no contexto escolar brasileiro aos problemas relacionados aos erros na escrita, esses podem estar na raiz das causas que explicam muitos casos de déficits de aprendizagem como elucidado, em seu estudo, por (FEDER \& MAJNEMER, 2007), "Simner descobriu que os erros de formulário, definidos como adições, exclusões ou desalinhamentos, no jardim da infância preveem as habilidades acadêmicas posteriores de leitura e de escrita das crianças.". O ensino compartimentalizado que ainda prevalece no sistema educacional brasileiro, em que os anos de estudos e as disciplinas, quase nunca comunicam objetivos, deixando à alfabetização e o desenvolvimento da escrita a cargo exclusivo das séries iniciais e de disciplinas específicas, pode dificultar a superação de baixo rendimento no desenvolvimento de habilidades importantes, já que, segundo a citação de (FEDER \& MAJNEMER, 2007) "Sandler et al. descobriu que crianças com transtornos de escrita tinham uma tendência para o baixo desempenho matemático, baixo QI verbal e as dificuldades de atenção aumentam em comparação com os controles." Isto pode levar a conclusão de que há uma forte relação possível entre problemas de escrita e outros déficits de aprendizagem, mas por ser complexa e envolver a interação de vários elementos, a identificação das causas dos problemas de escrita é dificultada em vários casos, segundo (FEDER \& MAJNEMER, 2007), "A razão subjacente da dificuldade de escrita 
de uma criança muitas vezes pode não ser clara, como elementos do processo de escrita estão estreitamente interligadas, por exemplo, dificuldade em ortografia pode contribuir para a dificuldade de anotações ou expressão de ideias". A complexidade inerente à atividade da escrita e o fato de cada pessoa responder de forma diferente aos desafios impostos evidencia a necessidade de instrumentos mais eficazes e adequados às peculiaridades dos indivíduos que apresentam problemas de caligrafia.

O produto da escrita é fruto do alinhamento dinâmico de fatores cognitivos, perceptivos e motores e traduz-se na forma mais usual de avaliação do desempenho escolar da criança e por este motivo, a má qualidade da escrita pode comprometer a identificação das capacidades do aprendente, o que pode ser fundamentado na afirmação de (FEDER \& MAJNEMER, 2007) "Está claro que as dificuldades de escrita podem ofuscar as capacidades de uma criança em outras áreas, fazendo com que o sucesso na escola seja menos facilmente atingível.". Atenuar os efeitos da disgrafia é de suma importância para garantir um desenvolvimento escolar pleno, permitindo que todos os potenciais do aprendente sejam evidenciados e estimulados de maneira adequada através de atividades condizentes com as limitações identificadas, propondo alternativas que, no mínimo minorem os efeitos do déficit de caligrafia quando o mesmo não puder ser totalmente corrigido através de abordagens de remediação.

\section{Desenvolvimento da Escrita}

Escrever é uma tarefa complicada e exaustiva que exige o máximo de concentração das crianças, principalmente nas primeiras fases de aquisição desta habilidade no início da vida escolar dos aprendentes, segundo (FEDER \& MAJNEMER, 2007), "A caligrafia é uma habilidade perceptivo-motora complexa que engloba uma mistura de habilidades visual-motora de coordenação, planejamento motor, cognitivo e habilidades de percepção, bem como de sensibilidades tátil e cinestésica". Esta constatação nos leva a crer que uma atenção especial deve ser dada ao desenvolvimento da motricidade fina durante os anos de educação infantil com sistematização das tarefas próprias da pré-escola que visem o desenvolvimento das habilidades motoras nos períodos que compõem o início do processo formal de aquisição da escrita na escola.

O aprendizado da escrita dá-se por fases de aproximações sucessivas do domínio dessa habilidade e a observação criteriosa, passo-a-passo do seu desenvolvimento pode nos fornecer indícios de distúrbios ainda em fases precoces, o que permitiria aos pais de escolares e educadores, com o amparo de diagnósticos emitidos por profissionais habilitados, lançar mão de estratégias de remediação e atenuação dos efeitos de problemas com a grafia, vejamos em (VAN HOORN, MAATHUIS, \& HADDERS-ALGRA, 2013) que: "Semelhante ao desenvolvimento da linguagem oral, a aquisição da capacidade de escrita progride por fases". Os estágios de desenvolvimento da escrita estão bem documentados mostrando que "Na idade de 2 anos, a criança começa a desenhar formas geométricas que começam com traços verticais. Traços horizontais seguem com a idade de 2 anos 
e meio e os círculos com a idade de três." (VAN HOORN et al., 2013). Aos 4 anos e até os 5 anos e meio os desenhos vão se tornando mais intencionais e conscientes, permitindo que a criança faça cópias de formas geométricas e outros desenhos nos quais ela já consegue ver significado indicando uma prontidão para escrita como afirma (VAN HOORN et al., 2013) "A capacidade da criança para copiar formas geométricas, em especial a cruz oblíqua, é vista como uma indicação de prontidão para a escrita". Conclui-se, então que é possível perscrutar, ainda bem cedo o potencial das crianças para a escrita.

É comum ver, em casas com crianças pequenas, paredes e móveis rabiscados, isto ocorre porque a escrita nos primeiros anos de vida manifesta-se como uma tentativa de converter em símbolos impressos um tipo de comunicação que a criança tenta estabelecer com o ambiente, como podemos conferir, também em (VAN HOORN et al., 2013), "Estes rabiscos ou escrita precoce refletem o seu entendimento de que a escrita serve a uma função simbólica, ou seja, sequências de símbolos representam sequências de unidades linguísticas.". A predisposição genética parece explicar a tendência para a comunicação escrita assim como explica a predisposição para aquisição da linguagem oral.

Essas tentativas de expressão através de desenhos podem ser consideradas como um primeiro estágio, ainda instintivo da escrita que deverá evoluir para experiências mais intencionais e cada vez mais estruturadas, (FEDER \& MAJNEMER, 2007) afirmam que: "O desenvolvimento da escrita começa com rabiscos precoces, o que torna-se mais intencional com o tempo. A medida que a criança se desenvolve, padrões de projeto evoluem para formas mais precisas e, em seguida letras. Formas das letras muitas vezes podem ser vistas em desenhos infantis que podem ser entendidos como um aprendizado para escrita". É imprescindível, para que este desenvolvimento seja facilitado, que haja acompanhamento e heterogestão pedagógica adequada ao estágio de desenvolvimento da aprendizagem da criança.

A pronúncia clara evidenciando todos os contornos da palavra dirigida ao bebê em fase da aquisição da linguagem oral é de extrema importância para identificação e apreensão da palavra pela mesma, como vemos em (CUTLER \& BUTTERFIELD; VAN DE WEIJER citado por VAN HOORN ET AL., 2013), "Para complicar ainda mais, a maioria da fala dirigida aos bebês consiste em expressões múltiplas de palavras para as quais faz-se pausas, que são as pistas acústicas mais salientes, o que não sinaliza de forma confiável limites de palavra". Por analogia, então, pode ser possível concluir, também, que é de extrema importância que a letra e consequentemente a palavra escrita ofereça todas as pistas gráficas necessárias à identificação e apreensão do grafema pelo aprendente da linguagem escrita. Para que este possa ser capaz de reproduzi-la ao tempo em que se apropria de seu significado e função, usa estratégias que o possibilita entender o que ainda é desconhecido de forma análoga ao que acontece com a linguagem oral como explicitado por (CUTLER \& BUTTERFIELD; VAN DE WEIJER citado por VAN HOORN et al., 2013) "(...) lactentes pré-verbais dificilmente podem contar com o conhecimento semântico-lexical; uma vez que precisam gerar candidatos lexicais bottom-up a partir de características acústicas e de distribuição 
da entrada de fala". Os contornos, forma solta como as letras de forma são grafadas facilitam a cópia de palavras com a identificação de cada letra que as compõem, além disso, há uma incidência muito maior desse tipo de letra no dia-a-dia, seja nas telas dos computadores, smartphones, tabletes, revistas, jornais e livros, então é possível encontrar justificativas plausíveis para o uso dessa tipologia de grafema na fase inicial de ensino da escrita na pré-escola e início do primeiro ano do ensino fundamental e gradativamente migrar para a escrita cursiva respeitando o desenvolvimento da motricidade fina e a evolução da capacidade de reconhecimento de textos da criança, essa abordagem já é usada em alguns países como podemos ver na citação a seguir: "Nos Países Baixos, em geral, as crianças são primeiramente ensinadas a escrever em letra de imprensa e avançar mais tarde para escrita cursiva" (VAN HOORN et al., 2013). No entanto faz-se necessário empreender estudos sobre o assunto, a fim de comprovar, ou não a maior eficácia para aquisição da escrita formal do uso inicial das letras de forma na alfabetização com a implementação gradativamente da escrita cursiva aliada à observação de outros fatores extrínsecos relacionados à escrita como podemos ver em (FEDER \& MAJNEMER, 2007) “(...)altura da cadeira / mesa, instrumento usado ao escrever, tipo de papel utilizado e sua colocação sobre a mesa, iluminação e som ambiental, distância quadro negro quando há cópia, e o volume de escrita que espera-se que a criança conclua". A adaptação do mobiliário e das ferramentas utilizadas na escrita ainda, em muitos casos prescinde de uma atenção maior no que diz respeito à ergonomia e demais variáveis ambientais.

\section{Disgrafia}

A etimologia da palavra disgrafia remete-nos ao grego antigo e corresponde a junção das palavras "dis" e "ghaphia" que significam respectivamente "dificuldade ou privação" e "escrita manual", um outro conceito baseado nas características da disgrafia pode ser visto em (NICOLSON \& FAWCETT, 2011) que asseveram que a "disgrafia é caracterizada por dificuldades na escrita."

O Manual Diagnóstico e Estatístico de Transtornos Mentais (DSM-IV) caracteriza a disgrafia como sendo:

uma aptidão para a escrita (medida por provas normalizadas, aplicadas individualmente, ou por uma avaliação funcional das aptidões de escrita) que se situa substancialmente abaixo do nível esperado para a idade cronológica do sujeito, quociente de inteligência e escolaridade própria para a sua idade.

Pode-se classificar a disgrafia, ainda de duas maneiras (perceptiva e motora) a criança que apresenta disgrafia perceptiva não consegue estabelecer a relação entre a simbologia e os grafemas que representam a fala ou outros sons, as palavras e frases; na disgrafia motora (discaligrafia), a criança consegue falar e ler, mas tem dificuldades na coordenação motora fina para escrever, ela percebe visualmente o texto, mas não consegue fazer os movimentos próprios da escrita. Engel-yeger, Nagauker-yanuv, \& Rosenblum (2009) citam Hamstra-Bletz and Blote (1993) que conceituam a disgrafia "como um distúrbio ou dificuldade na produção da linguagem escrita 
relacionada com a mecânica da escrita", ou seja, quando a capacidade de escrever é pobre ou a letra é ilegível, vê-se nisso um indício de disgrafia.

O estudo sobre a disgrafia avançou consideravelmente a partir das descobertas científicas impulsionadas principalmente pela análise de pacientes com danos cerebrais cuja sintomatologia incluía comprometimentos da escrita, segundo (IIJIMA, 1991) "Danos cerebrais em pacientes com transtornos adquiridos de escrita fornecem informações importantes sobre os processos normais de ortografia e escrita". Os pesquisadores e demais estudiosos das ciências da educação ainda precisam agregar grande parte desses achados aos seus referenciais de estudo, aumentando, assim a possibilidade de uma maior aproximação entre as descobertas das Neurociências e o corpo de saberes relativos à Pedagogia e demais ciências que tem no processo educacional um campo de estudo a explorar.

\section{Disgrafia Decorrente de NF1 e de Mal de Parkinson}

A alteração de um único gene mais comum a afetar o sistema nervoso humano é conhecida como neurofibromatose tipo 1 (NF1) e é caracterizada segundo (GILBOA, JOSMAN, FATTALVALEVSKI, TOLEDANO-ALHADEF, \& ROSENBLUM, 2010) “(...)por sinais neurocutâneas, com manchas cor de cafe'-com-leite, sardas axilares, neurofibromas cutâneos e hamartomas de íris (nódulos de Lisch) que ocorrem na maioria dos pacientes." A NF1 também é “(...) uma doença multisistémica, com uma vasta variedade de manifestações oculares, do esqueleto, do sistema endócrino, e dos vasos sanguíneos, assim como dos sistemas nervosos central e periférico". Seria possível deduzir que as manifestações ocuralares devem ser responsáveis pela alteração na visopercepção que é um dos fatores subjacentes da qualidade da escrita. No entanto na conclusão do estudo de (GILBOA et al., 2010) eles sugerem que há "(..) possibilidade de uma relação entre dificuldades relacionadas as funções executivas e o mau desempenho na escrita." Também alertam para o fato de que uma "atenção e intervenção precoce de professores e pais nas dificuldades de caligrafia de crianças com diagnóstico de NF1, podem influenciar muito o seu desempenho acadêmico global, funcionamento social e bem-estar emocional". Isso reforça a indicação de que a disgrafia pode afetar o desenvolvimento da criança no que se refere à aquisição e incremento de outras habilidades.

A escrita é uma tarefa comum no dia-a-dia e problemas relacionados a ela são rapidamente percebidos por quem desenvolve algum distúrbio de caligrafia, o que acaba sendo uma das causas que levam as pessoas com Doença de Parkinson (DP) a procurarem um médico como afirma (LETANNEUX, DANNA, VELAY, VIALLET, \& PINTO, 2014) "Distúrbios da caligrafia não são formalmente incluídos no diagnóstico da doença de Parkinson (DP). No entanto, é muitas vezes um dos sinais iniciais que levam o paciente a consultar-se." A caligrafia acaba sendo afetada em pacientes com DP, pois é segundo (LETANNEUX et al., 2014) “(...) uma atividade motora fina generalizada que exige grande perícia e forte precisão." Enquanto a DP "afeta ambos os 
movimentos voluntários e automáticos". Isso torna os problemas de escrita idícios consideráveis para o diagnóstico da DP.

A disfunção de escrita mais comum em pacientes com DP é a micrografia, segundo (Letanneux et al., 2014) "Micrographia é uma redução anormal no tamanho da escrita e é um déficit comportamental específico associada à DP." Essa disfunção geralmente precede outras disfunções motoras nos pacientes com DP.

\section{Avaliação da Escrita}

A avaliação para identificar causas e níveis de distúrbios que impactam o desenvolvimento adequado da escrita é de essencial importância na garantia do sucesso escolar, dado o significado desta habilidade principalmente nos primeiros anos do ensino fundamental, essa importância é evidenciada por (BISHOP \& CLARKSON, 2003) quando afirmam que: "A capacidade de se expressar através da escrita é uma habilidade de crucial importância nas sociedades letradas, e ainda a avaliação da linguagem escrita é muitas vezes esquecida quando se avalia crianças que apresentam problemas de comunicação". Garantir que não haja eventuais perturbações nas características intrínsecas e extrínsecas de um dado fenômeno é essencial para que se possa avaliá-lo de forma mais fiável e isso não seria diferente nos casos de avaliação da disgrafia, e para isso a avaliação deve ser feita preservando as condições em que o fenômeno ocorre na realidade, segundo (VAN HARTINGSVELDT, DE GROOT, AARTS, \& NIJHUIS-VAN DER SANDEN, 2011) "Uma tendência recente em terapia ocupacional é concentrar a avaliação em situações da vida real, tornando a avaliação de desempenho contextual e significativa." Considerar as reais condições em que a escrita é desenvolvida cotidianamente garante ao processo avaliativo a preservação de variáveis que poderiam mudar totalmente o resultado da avaliação caso fossem alteradas.

Minimizar os efeitos da disgrafia requer a identificação precoce das crianças que apresentam distúrbio na escrita, segundo (VAN HARTINGSVELDT et al., 2011) "Os efeitos negativos das dificuldades de escrita sobre o desempenho acadêmico e autoestima de uma criança, como discutido na literatura, torna de grande importância fazer a avaliação precoce de habilidades relacionadas à pré-escrita.". Também um diagnóstico individualizado é de extrema importância, já que cada criança responde de maneira própria às variáveis ambientais, como podemos comprovar na citação de (VAN HARTINGSVELDT et al., 2011) "Cada criança reage de maneira diferente às variáveis ambientais; o que pode melhorar a performance em uma criança pode ser uma barreira para o desempenho em outra." Isso se deve ao grau de maturação e características biopsicossociais que são peculiares a cada indivíduo.

No campo da avaliação da escrita, ainda há muito a fazer, já que a disponibilidade de instrumentos confiáveis para avaliação da qualidade da escrita é recente, segundo (FEDER \& MAJNEMER, 2007) “Em uma pesquisa cross-Canadá de terapeutas ocupacionais, raramente foram utilizados avaliações formais de escrita, possivelmente refletindo a falta de disponibilidade, no 
momento de uma ferramenta de avaliação de escrita válida e confiável.". Hoje já há um número razoável de instrumentos de avaliação sendo utilizados.

$\mathrm{Na}$ condução dos testes de escrita é necessário considerar as diferenças entre o texto espontâneo criado pela própria criança no momento da escrita, a cópia de textos previamente impressos ou a escrita a partir de texto ditado, segundo (JANG, KIM, PARK, \& LEE, 2015) "Finalmente, num único teste de cópia, nossos resultados mostraram que a negligência unilateral espacial foi significativamente relacionada com disgrafia. Ela pode ser causada por que a cópia precisa de mais processamento visuoperceptivo do que a escrita espontânea ou de testes de ditado.". Assim, percebe-se que as diferentes tarefas relacionadas à escrita envolvem níveis de complexidade distintos.

\section{Instrumentos de Avaliação da Escrita}

A escolha do instrumento de avaliação deve considerar as peculiaridades do sujeito a ser avaliado, segundo (FEDER \& MAJNEMER, 2007) "O instrumento escolhido deve corresponder melhor a área de dificuldade na escrita da criança, de modo a facilitar a implementação de uma estratégia de tratamento eficaz." ao escolher o instrumento é preciso que o profissional tenha ciência das características do mesmo, conhecendo os seus potenciais e limitações como podemos ver, também em (FEDER \& MAJNEMER, 2007) "Na seleção de uma ferramenta de avaliação, é importante que o médico tenha conhecimento das propriedades psicométricas do instrumento, tendo em mente os seus pontos fortes e limitações". Ver-se-á que os instrumentos de avaliação se destinam à áreas específicas relacionadas à escrita.

A forma mais comum e tradicional de avaliação da escrita é através de textos produzidos usando apenas papel e lápis, em seu estudo (VAN HARTINGSVELDT et al., 2011) apresentam dois instrumentos para avaliação da escrita com tarefa papel e lápis, Scale of Children's Readiness in Printing (SCRIPT) e Draw-A-Person: Quantitative Scoring System (DAP:QSS). O SCRIPT é descrito segundo os autores como“(...) um teste de cópia de letra de forma desenvolvido para crianças do jardim da infância. A criança tem que copiar todas as 26 letras minúsculas e oito letras maiúsculas, ou seja, $A, K, M, N, V, W, Y$, e Z. Já o DAP:QSS “(...)é uma avaliação de desenho figuras com um sistema de pontuação quantitativa.". Os dois testes permitem avaliar a fase da pré-escrita.

Um outro teste de escrita utilizando papel e lápis é o Writing Essential Skill ScreenerPreschool version WESS-P que permite fazer uma avaliação da prontidão para escrita ao nível da integração visomotora, segundo (VAN HARTINGSVELDT et al., 2011) "O WESS-P consiste em quatro atividades que refletem as habilidades de pré-escrita e desenvolvimento conceitual e inclui: copiar formas geométricas simples, a velocidade de cópia, copiar letras e números, e escrita de nome.". Os autores apresentam apenas esse instrumento de avaliação para prontidão da escrita a partir da integração visomotora. 
Quanto a coordenação motora fina levantamos a existência de dois instrumentos o Test of In-Hand Manipulation-revised (TIHM-R) e o Nine Hole Peg Test (NHPT) que segundo (van Hartingsveldt et al., 2011) "Ambos os testes utilizam um procedimento de tempo com pegboard e pinos. No TIHM-R, os pinos devem ser usados em tarefas de manipulação com a mão, como translação e rotação com estabilização;" sobre o NHPT (VAN HARTINGSVELDT et al., 2011) evidenciam que "os pinos devem ser utilizados em uma tarefa que visa apenas uma mão.", neste caso a mão utilizada para escrever.

Sobre a integração visomotora temos um teste apresentado por (VAN HARTINGSVELDT et al., 2011), trata-se do Developmental Test of VisualMotor Inte- gration (Beery VMI) segundo (Gilboa et al., 2010) "Este teste é adequado para discernir os diferentes processos envolvidos nas tarefas de coordenação mão-olho (por exemplo, de escrita), percepção visual, integração visual-motora, e os processos de coordenação motora.." O teste consistiria de uma tarefa de integração visomotora na qual segundo (GILBOA et al., 2010) “(...)crianças são convidadas a copiar 24 figuras geométricas começando com figuras simples e terminando com as mais complexas.". Pode-se listar ainda cinco instrumentos para testar a coordenação motora de forma geral:

Motor Performance Checklist (MPC); (9) Movement Assessment Battery for Children, 2nd edition (M-ABC-2); (10) Maastricht's Motor Test (MMT); (11) Bruininks-Oseretsky Test of Motor Proficiency, 2nd edition (BOT-2); e (12) the Denver Developmental Screenings Test, 2nd edition (Denver-II) (GILBOA et al., 2010)

O surgimento de ferramentas computadorizadas permitiu a identificação de fatores relacionados à escrita até então de difícil detecção como o tempo em que o lápis passa em contato com o papel ou suspenso no ar, a variação de pressão do lápis contra a superfície, velocidade da escrita em diferentes trechos do texto, além de facilitar a avaliação de conformidade do texto produzido com um dado padrão textual. (ROSENBLUM et al., 2003 citado por GILBOA et al., 2010) fazem referência a ferramenta computadorizada Computerized Penmanship Evaluation Tool (ComPET): "Essa avaliação de escrita padronizada e validada utiliza uma mesa digitalizadora e software on-line de coleta e análise de dados. Ele foi desenvolvido para efeitos de recolha de medidas objetivas do processo de escrita manual" e independe da língua, sendo possível utiliza-lo em qualquer idioma.

\section{Remediação da Escrita}

A remediação da escrita deve levar em consideração tanto os fatores intrínsecos como os fatores extrínsecos, adequar o ambiente observando os aspectos ergométricos podem ajudar a melhorar a escrita como vemos em (FEDER \& MAJNEMER, 2007) "O ideal é que a criança esteja sentada com os pés apoiados no chão, quadris e região lombar apoiada para trás contra a cadeira, joelhos flexionados a aproximadamente $90^{\circ}$ e cotovelos levemente flexionados com antebraços descansando confortavelmente na superfície da mesa". Aspectos simples como a adequação da altura da mesa e da cadeira às características de quem as utiliza durante a escrita podem fazer a 
diferença positiva na qualidade da escrita, segundo (FEDER \& MAJNEMER, 2007) "A mesa alta ou a cadeira muito baixa irá incentivar a curvatura para a frente e, inversamente, quando a mesa é baixa e a cadeira demasiado elevada e / ou pés sem apoio, a saída da escrita pode ser comprometida.", ajustes de adequação às medidas da criança podem facilitar a produção da escrita com melhoria da qualidade da letra.

A reabilitação da escrita, ainda deve considerar uma séries de fatores, segundo (FEDER \& MAJNEMER, 2007) "As abordagens de tratamento documentadas sobre a escrita manual na literatura, incluem intervenções individualizadas / exercícios perceptivo-motor, visual-motor, controle motor e instrução de escrita manual complementar.." Mas os próprios autores fazem uma ressalva sobre a falta de um maior número de estudos sobre o assunto, "No entanto, existem poucos estudos controlados que examinaram abordagens de tratamento específicos de intervenção para escrita manual, usando um tamanho da amostra maior". (FEDER \& MAJNEMER, 2007). Vemos, então um campo propício a condução de mais estudos para elucidar-se as formas de intervenção reabilitadoras da escrita, bem como conhecer abordagens didáticas mais eficazes no desenvolvimento dessa habilidade.

\section{CONCLUSÃO}

As descobertas das Neurociências podem trazer contribuições valiosas para as práticas pedagógicas, ajudando a ampliar a compreensão sobre os sintomas, causas e consequências de diversos déficits de aprendizagem, bem como poderia auxiliar na busca de meios para remediar, minorar ou, até mesmo eliminar os efeitos de tais déficits, contribuindo assim, com a garantia de uma vida escolar com possibilidade de pleno desenvolvimento, cognitivo, afetivo e psicomotor para um contingente considerável de crianças que sofrem de distúrbios limitadores de seu potencial de aprendizagem como, é o caso da disgrafia que limita a habilidade de escrever, segundo (FEDER \& MAJNEMER, 2007) "A competência da escrita não é apenas importante para o sucesso acadêmico em idade escolar, mas é, também uma habilidade crítica em toda vida adulta.". A habilidade da escrita, como já tratou-se neste estudo é uma ocupação complexa, na qual estão envolvidos múltiplos fatores, tanto inerentes ao sujeito que escreve quanto ao ambiente onde a escrita é desenvolvida, ainda segundo (FEDER \& MAJNEMER, 2007) "Apesar das dificuldades da escrita manual poderem ser o resultado de fatores intrínsecos, como habilidades e componentes pobres, considerações extrínsecas, como o ambiente acadêmico e a biomecânica não devem ser esquecidas.". Os autores (FEDER \& MAJNEMER, 2007), também chamam a atenção para a importância de profissionais das áreas da educação e da saúde ficarem atentos às consequências de uma caligrafia ruim, asseverando que essas podem ser de grande alcance: "É especialmente importante que os profissionais de saúde e educadores compreendam as consequências acadêmicas e psicossociais de grande alcance da má caligrafia.", essas consequências podem significar o insucesso escolar ou profissional do sujeito. 
O uso da língua confere status social, quando a utilizamos de acordo com os padrões cultos de forma clara e polida esse status eleva-se, quando, ao contrário não somos capazes de articulála de forma inteligível, ferindo os padrões estabelecidos para a mesma, somos avaliados de forma negativa o que afeta a nossa autoimagem e põe em dúvida a nossa capacidade de superar outros desafios relacionados ao domínio da língua, e isso é verdade para qualquer um dos suportes em que a língua é usada.

\section{REFERÊNCIAS}

BISHOP, D. V. M.; CLARKSON, B.. Written language as a window into residual language deficits: a study of children with persistent and residual speech and language impairments. Cortex; a Journal Devoted to the Study of the Nervous System and Behavior, v.39, n.2, p.215-237. (2003). DOI: http://doi.org/10.1016/S0010-9452(08)70106-0

\section{ENGEL-YEGER, B.; NAGAUKER-YANUV, L.; ROSENBLUM, S.. Self-Effi cacy Among Children With Dysgraphia, v.63, n.2, 2009.}

FEDER, K. P.; MAJNEMER, A.. Handwriting development, competency, and intervention. Developmental Medicine and Child Neurology, p.312-317, 2007. DOI: http://doi.org/10.1111/j.1469-8749.2007.00312.x

GILBOA, Y.; JOSMAN, N.; FATTAL-VALEVSKI, A.; TOLEDANO-ALHADEF, H.; ROSENBLUM, S.. The handwriting performance of children with NF1. Research in Developmental Disabilities, v.31, n.4, p.929935, 2010. DOI: http://doi.org/10.1016/j.ridd.2010.03.005

IIJIMA, S.. Helical microtubules of graphitic carbono. Nature, n.354, p.56-58, 1991. DOI: http://doi.org/10.1038/354056a0

JANG, D.; KIM, M.; PARK, K. H.; LEE, J. W.. Language-specific Dysgraphia in Korean Patients with Right Brain Stroke : Influence of Unilateral. Spatial Neglect, 323-327. (2015).

LETANNEUX, A.; DANNA, J.; VELAY, J.-L.; VIALLET, F.; PINTO, S.. From micrographia to Parkinson's disease dysgraphia. Movement Disorders, 29(12), 1467-1475. (2014). DOI:

http://doi.org/10.1002/mds.25990

MARKMAN, T. M.; QUITTNER, A. L.: EISENBERG, L. S.; TOBEY, E. A.: THAL, D.; NIPARKO, J. K.; WANG, N. Y.. Language development after cochlear implantation: An epigenetic model. Journal of Neurodevelopmental Disorders, 3(4), 388-404. (2011) DOI: http://doi.org/10.1007/s11689-011-9098-z

NICOLSON, R. I.; FAWCETT, A. J.. Dyslexia, dysgraphia, procedural learning and the cerebellum. Cortex, 47(1), 117-127. (2011) DOI: http://doi.org/10.1016/j.cortex.2009.08.016

VAN HARTINGSVELDT, M. J.: DE GROOT, I. J. M.: AARTS, P. B. M.; NIJHUIS-VAN DER SANDEN, M. W. G.. Standardized tests of handwriting readiness: A systematic review of the literature. Developmental Medicine and Child Neurology, 53(6), 506-515. (2011) DOI: http://doi.org/10.1111/j.1469$\underline{8749.2010 .03895 . x}$

VAN HOORN, J. F.; MAATHUIS, C. G. B.; HADDERS-ALGRA, M.. Neural correlates of paediatric dysgraphia. Developmental Medicine and Child Neurology, 55(SUPPL.4), 65-68. (2013). DOI: http://doi.org/10.1111/dmcn.12310 